\title{
Software-Defined Wireless Mesh Networking: Current Status and Challenges
}

\author{
Michael Rademacher*, Karl Jonas, Florian Siebertz, Adam Rzyska, \\ Moritz Schlebusch ANd Markus Kessel \\ Department of Computer Science, University of Applied Sciences Bonn-Rhein-Sieg, Sankt Augustin, \\ Germany \\ *Corresponding author: michael.rademacher@h-brs.de
}

\begin{abstract}
The combination of Software-Defined Networking (SDN) and Wireless Mesh Network (WMN) is challenging due to the different natures of both concepts. SDN describes networks with homogeneous, static and centralized controlled topologies. In contrast, a WMN is characterized by a dynamic and distributed network control, and adds new challenges with respect to time-critical operation. However, SDN and WMN are both associated with decreasing the operational costs for communication networks which is especially beneficial for internet provisioning in rural areas. This work surveys the current status for Software-Defined Wireless Mesh Networking. Besides a general overview in the domain of wireless SDN, this work focuses especially on different identified aspects: representing and controlling wireless interfaces, control-plane connection and topology discovery, modulation and coding, routing and load-balancing and client handling. A complete overview of surveyed solutions, open issues and new research directions is provided with regard to each aspect.
\end{abstract}

Keywords: software-defined networking; wireless mesh networks; wireless backhaul; OpenFlow; routing; bootstrapping; WiFi; IEEE 802.11

Received 30 November 2016; revised 28 April 2017; editorial decision 5 June 2017

Handling editor: Linghe Kong

\section{INTRODUCTION AND MOTIVATION}

Software-Defined Networking (SDN) and OpenFlow (OF) started as an academic experiment but emerged to a paradigm that gives hope to change the limitations of current static network infrastructures [1]. 'SDN is a conceptual framework in which networks are treated as abstractions and are controlled programmatically, with minimal direct touch of individual network components' [2]. SDN tries to fulfill a need to configure network devices more flexible and dynamically. This is achieved by taking away the process of making decisions about packet handling from every single device. Instead, a logically centralized controller entity is deployed to make these decisions for all devices in the entire network [1]. Overall, this leads to a paradigm shift from monolithic devices which combine control, monitoring, management and data-forwarding functions in a single entity toward a clear separation of control and data-plane [3]. SDN has been identified to further decrease the Capital Expenditure (CAPEX) and Operational Expenditure (OPEX) for Internet Service Provider (ISP) [4]. In wired networks, commercial roll-out has begun.

A Wireless Mesh Network (WMN) is considered as a multi-hop wireless network, in which mesh nodes relay traffic on behalf of other mesh nodes or connected clients (and networks) and thereby form a wireless backbone [5]. In the initial design of a WMN, wireless mesh routers were equipped with only one radio and a single channel was used for communication [6]. Multiple performance evaluations of this single-radio approach were conducted and researchers showed that the available access capacity for each node significantly decreases with the network size [6]. Dual-radio mesh networks were proposed where one radio is dedicated to access and one to forward packets. However, in this architecture, adjacent mesh links still use the same channel and are therefore prone to interference issues which lead to 
performance degradation. This issue has been addressed with the proposal of multi-radio multi-channel WMNs (MR-MC WMNs) where, in the best case, interference free channels are assigned to each radio. These MR-MC WMNs have been used in a variety of different applications [6]. In the context of internet provisioning in rural areas, an evaluation of MRMC WMNs has been proposed by Ref. [7]. The author labeled this emerging network class as Wireless Backhaul Network (WBN) and a formal definition is summarized as follows. In a hierarchical telecommunication network, the WBN comprises the intermediate links between the core of the network and the sub-networks at the edge, using a costefficient wireless transmission technology (mainly 802.11). Coordinated WBNs usually consist of stationary nodes and exhibit characteristics of carrier-grade networks. Links in WBNs are point-to-point connections between two mesh interfaces and operate over long-distances using directional antennas. An operational example of a WBN is provided in Ref. [3]. WBN have been identified to decrease the CAPEX and OPEX for Wireless Internet Service Provider (WISP) [8].

Our motivation is a joint approach of SDN and WBN for cost-efficient internet provision in rural areas. Benefits of such a combination are:

- If SDN is already utilized in the core part of a network, it feels natural to extend it further into the last miles. This provides ISP toward the possibility of holistic network management and control.

- SDN maintains a centralized network state. This provides the flexibility to configure, manage, secure and optimize the network resources using exchangeable applications [2].

- In WMN, traffic pattern can change on a relatively small time-scale. With a centralized SDN-based structure, the network can react to these changes based on global knowledge.

- With an increased number of nodes in the network, multiple gateways providing interconnection to other networks become mandatory. SDN applications can handle these multiple gateways and configure the flows accordingly [9].

- SDN can be maintained with minimal effort at a centralized instance. The minimal direct touch of individual network components reduces the need for experts in the field and provides the possibility to simply exchange components in case of (hardware) failures [10].

- A common advantage of SDN is the goal to deploy standardized, vendor-independent hardware wherever possible, leading to higher competition and lower cost.

However, a combined SDN and WMN is a challenging task due to the different natures of both concepts [11]. SDN describes networks with homogeneous, static and centralized controlled topologies. In contrast, a WMN is characterized by a dynamic and distributed network control. For simplicity, we will refer to the combination of SDN and WMN or WBN as Software-Defined Wireless Mesh Network (SDWMN). SDWMN define the main topic of this work.

There exist various publications offering comprehensive overviews about SDN in the wired domain, covering the main concepts, distinctive features and possible future research directions $[1,12]$. WMN have been a research topic for more than two decades [13]. Several comprehensive and well-regarded literature reviews have been published [13, 14, 15]. These reviews provide a general overview of various topics and together show the development in this research field. The authors in [15] provide a comprehensive list of specific aspects researchers have already addressed in WMN.

Initial discussions about the applications and different aspects of wireless SDN are provided in $[17,16,18]$. At the moment, researchers start to investigate even particular types of wireless SDN for example Software-Defined Wireless Sensor Network (SDWSN) [19]. Most related to our work, Ref. [20] presents a broader overview about SDN in the wireless domain in general. The authors address several contexts like Wireless Cellular Network, WSN, Wireless Home Network and WMN. In the area of combined SDN and WMN approaches, the authors briefly summarize current projects discussing aspects like fault tolerance or energy efficiency.

Compared to previous publications, this work identifies and focuses on five fundamental aspects for SDWMN. These are specifics of the wireless interface, control-plane connection and topology discovery, routing and load-balancing, modulation and coding, and client handling. They are important in various applications but especially for our motivated use-case of WBN. For each aspect, we discuss existing solutions from different domains and provide ideas for future research directions. Therefore, the idea of this work is not to function as a blueprint for SDWMN but rather to evaluate various different possibilities.

The rest of this work is structured as follows: In Section 2, we provide a general overview on recent work on wireless SDN especially focusing on the inclusion of different technologies. The idea of this section is to provide a broader view on this domain before solely focusing on SDWMN. Section 3 is the main part of this work. In this section, we present the different aspects for SDWMN in individual parts. The structure for each aspect is the same. First, the aspect is described in detail. Second, previous publications addressing this particular aspect are summarized and discussed. Third, open issues and future research ideas are provided. This work closes with a summary and conclusion in Section 4.

\section{WIRELESS SDN}

The purpose of this section is to provide a general overview of SDN in combination with various wireless technologies. 
Its methodology follows a top-down structure, narrowing down related work to SDWMN.

SDN in wired networks has been the topic of a large variety of work, examining even specific problems such as security or Quality of Service (QoS) in great detail. The most prominent implementation of SDN is OF. At the moment, many vendors of networking infrastructure have started to incorporate $\mathrm{OF}$ in their equipment. Also, in the academic world, $\mathrm{OF}$ is by far the most dominant SDN protocol. Therefore, we will use and refer to $\mathrm{OF}$ throughout this work. SDN in wireless networks on the other hand is just beginning to form itself on top of the experiences gained in the wired context. The main ideas of SDN do not change with using wireless links in order to transmit the data in the network. There is still a separation of control-plane and data-plane and a logically centralized controller manages the network's behavior. What is changed is the need for handling the new layer of complexity that is gained through the integration of wireless interfaces.

The idea of wireless SDN is discussed in two different ways in the research community. On the one hand, it is often mentioned in the context of architecture descriptions, where it describes a SDN controlled network in which the backbone is typically not wireless but interconnects several SDN controlled wireless access networks [21]. A typical example is an infrastructure network at a university. On the other hand, there are networks where the backbone itself is realized through SDN controlled wireless links [22]. The latter case corresponds to our view on SDWMN.

Some papers propose different, generic architectures to implement SDN into existing wireless technologies and networks from a rather high-level point of view. This is not limited to 802.11, but includes technologies like UMTS, LTE or WiMAX. In [21, 23], the basic SDN architecture is transferred to a carrier-grade network, spanning a core transport backbone and several wireless access networks. A centralized control-plane offers an abstraction of the network for QoS or authentication management, while enabling flow-level traffic shaping. The authors in [24] propose an architecture for dense wireless cells, where a central control-plane manages a heterogeneous access network as well as the backhaul. While the backhaul is controlled through typical SDN protocols like OF, separate protocols are utilized for fine-tuning the diverse access technologies regarding transmission power or frequency. To account for different levels of control in the network in terms of reaction time and level of detail, a vertical hierarchy of controllers is proposed.

In the context of mobile carrier networks, significant work has been conducted in [25]. The authors present a proof-ofconcept implementation to replace the Evolved Packet Core with a SDN-based framework called Software-Defined Mobile Networks. Similar work has been conducted in [26]. A comprehensive overview about recent work and future challenges for SDN in the mobile core network is provided in Ref. [27].
For further information on the general architecture and different use-cases of wireless SDN, we refer the reader to the survey conducted in [28].

One of the first publications which deals with 802.11 and SDN is OpenRoads [29]. The authors provide a blueprint for a campus-wide wireless access network. Their motivation is a wireless infrastructure for different experiments through isolated SDN slices. The authors make use of FlowVisor [30] to slice the data-path and SNMPVisor to slice the configuration.

The authors in [31] provide a detailed discussion about different projects to emulate or simulate wireless SDN. They propose that a lightweight virtualization with wireless channel emulation capabilities and mobility models can boost the research in the domain of wireless SDN experiments.

The authors in [32] present an architecture very similar to [33] which they call Odin and CloudMAC, respectively. But only the access part of the network utilizes wireless technology in their case and packets are forwarded between virtual Access Point (VAP) and stations based on Media Access Control (MAC) layer processing via $\mathrm{OF}$. This enables a flexible and centralized management of host connections, enabling further applications like dynamic spectrum use, on-demand scaling and improved roaming between AP. Additionally, it is proposed to include the 802.11 header into the list of headers that can be examined by OF. The authors in [34] propose an SDN architecture for dense WiFi deployments called Ethanol. The focus of their work is the definition of a WiFi capable southbound interface for getting and setting different parameters. Similar to [32], a server-agent application has been developed and the authors demonstrate different use-cases like load aware association control and mapping of flows to interface queues for QoS provisioning.

The work in [38] focuses on the combination of SDN and WMN. Particular thoughts are given on the architecture and the traffic orchestration. According to Ref. [38], the state-ofthe-art for SDWMN can be classified into two different categories: if the controller communicates in-band or out-ofband with the wireless switches. The authors in [28] describe basic use-cases for OF-based load balancing in a single-radio WMN. The authors make use of the Better Approach to MANET (B.A.T.M.A.N.) protocol to identify next-hop nodes and maintain the topology. On top, the authors use OF for load-balancing by monitoring connections and changing specific flows accordingly. A similar approach is presented in [22], using Optimized Link State Routing (OLSR) for setting up and maintaining a control network. The authors demonstrate their approach in the use-case of balancing traffic among multiple internet gateways with OF. The combination of OLSR and OF is also used in Ref. [5] to improve mobility management. Using well-known distributed mesh protocols for the control plane and SDN for the data-plane has been labeled as hybrid-approaches by Ref. [11].

The authors in [9] focus on Public Access WiFi Service-a community shared service technique to utilize unused capacity 
in home broadband connections by interconnecting routers in a WMN using SDN principles. The main part of the work is the evaluation of a traffic redirection algorithm to assign the optimum gateway to a particular user.

In [3], a concept for integrating SDN into a WBN infrastructure is presented which the authors call WiBACK. While this also covers a high-level point of view, a more detailed thought is given to how control- and data-plane must be adapted in order to provide the setup and configuration of diverse wireless interfaces on the network devices. Apart from the existing flowmanagement and monitoring, there are additional modules in the control-plane that gather information regarding the spectrum management and link capacity in the network via an extended southbound interface. Based on this knowledge, sophisticated configuration decisions can be applied. Further, the problem of initial configuration, for example when a node joins the network, is addressed. A minimal, local controller handles the connectivity of the node until the regular control entity is reached. In addition, the depicted architecture has been deployed and tested in various testbeds, showing the viability of the approach.

\section{ASPECTS FOR SDWMN}

While the last section provided a brief overview on the field of wireless SDN, we focus especially on important aspects for SDWMN in this section. Based on our experiences with WMN and WBN [42, 43, 44], we identified the following:

- Wireless interfaces in SDN,

- Control-plane connection and topology discovery,

- Routing and load-balancing,

- Modulation and coding,

- Client handling.

In the following, these aspects will be addressed in separate subsections; however, the methodology in each subsection is similar. First, the idea is described in more detail. Afterwards, we evaluate the current state-of-the-art and point out open issues and possible future research directions.

Table 1 provides an overview of different publications specifically addressing the network type SDWMN and how they incorporate the contemplated aspects. The idea of this table is to provide the reader with a quick overview of the current state-of-the-art before going into additional details. The reviews conducted in the following subsections are not limited to the work in Table 1. We added promising solutions and ideas from other wireless SDN domains as well to draw a complete picture for the defined aspects.

\subsection{Wireless interfaces in SDN}

Handling and configuring wireless interfaces are mandatory for sophisticated SDWMN. Since 802.11 is the main standard used in SDWMN at the moment, most of the examples in this section focus on this wireless technology. However, many ideas can be transferred to different wireless standards. In the wired world, a simple cable determines the connectivity between two devices. In addition, these wired links have a nearly constant physical capacity and no fluctuation due to environmental factors. Wireless links, however, require the configuration of a significant amount of parameters to establish a link. In the case of 802.11, these include the Service Set Identifier (SSID), the frequency and the transmission power. Therefore, it is important for SDWMN to provide a control mechanism for parameters related to wireless interfaces.

To be able to query and modify wireless interface parameters through SDN applications, it is necessary to expand the capabilities of the current SDN architecture. In theory, the requirements are 3-fold. First, Software running on the nodes provides basic features such as reading and modifying the current configuration parameters. Second, this software communicates over a protocol with another software on the controller's side. Third, the server-side software presents the nodes' configuration to applications in the controller and enables them to push new directives to the nodes. Despite this configuration aspect, an additional issue has been identified with 802.11 due to different addressing principles compared to Ethernet.

\subsubsection{Related work}

At the moment, there are different approaches discussed how the increased complexity of wireless interfaces should be controlled from a centralized SDN controller or if there is a need for such a control. A significant amount of publications do not include the wireless interface configuration in their SDWMN core architecture. Either no details about wireless configuration are provided or a static configuration is conducted and SDN principles run on top of it [36]. If all wireless interfaces are set to the same static parameters in an ad hoc mode, this leads to a maximum connectivity among nodes. However, this configuration significantly reduces the throughput and does not correspond to the state-of-the-art in WMN which makes use of a multi-radio multi-channel approaches.

Another widely regarded approach for the configuration of interfaces in SDWMN is using mesh protocols like OLSR, B. A.T.M.A.N. or $802.11 \mathrm{~s}$ to handle the configuration in a distributed manner [22, 37, 45]. The usage of SDN alongside a distributed mesh protocol on the wireless switches, is according to [11], a hybrid approach. However, the main idea of SDN is to decrease the complexity of the nodes as far as possible. A complete implementation of a mesh protocol on each node partially violates this idea. In addition, an important part of the network still functions in a non-SDN way.

The authors in [46] provide a different approach for the combination of SDN and WBN. The authors argue that the configuration of the wireless interfaces should not be exposed to the SDN controller. Instead, a modern distributed WMN 
TABLE 1. Overview of joint SDN and WMN approaches.

\begin{tabular}{|c|c|c|c|c|c|c|c|c|}
\hline & $\mathrm{OF}$ & Wirel. int. & Ctl-Conn. & Topo.-Disc. & $\mathrm{M} \& \mathrm{C}$ & Routing & Clients & Focus \\
\hline [5] & Yes & $\begin{array}{l}\text { Static but custom } \\
\text { monitoring }\end{array}$ & $\begin{array}{l}\text { Out-of-band } \\
\text { (SSID } \\
\text { segregation) }\end{array}$ & OLSR & No & $\begin{array}{l}\text { Client } \\
\text { distribution }\end{array}$ & Active & Hybrid architecture with custom monitoring \\
\hline$[35]$ & Yes & Static & $\begin{array}{l}\text { Out-of-band } \\
\text { (add. NIC) }\end{array}$ & $802.11 \mathrm{~s}$ & No & Chain & Wired & Experiments: WMN protocols vs. OF \\
\hline [36] & Yes & Static & $\begin{array}{l}\text { Out-of-Band } \\
\text { (add. NIC) }\end{array}$ & $802.11 \mathrm{~s}$ & No & Chain & Wired & Experiments: $802.11 \mathrm{~s}$ vs. OF \\
\hline [22] & Yes & Static & In-band & OLSR & No & $\begin{array}{l}\text { Gateway } \\
\text { selection }\end{array}$ & Passive & $\begin{array}{l}\text { Hybrid architecture and distribution of flows } \\
\text { among gateways }\end{array}$ \\
\hline [37] & Yes & Static & In-band (VPN) & $\begin{array}{l}\text { B.A.T.M. } \\
\text { A.N. }\end{array}$ & No & $\begin{array}{l}\text { Generic link } \\
\text { conditions }\end{array}$ & $\mathrm{N} / \mathrm{A}$ & $\begin{array}{l}\text { Hybrid architecture with dynamic load- } \\
\text { balancing }\end{array}$ \\
\hline$[38]$ & Yes & Extended-OF & $\begin{array}{l}\text { Out-of-band } \\
\text { (SDR) }\end{array}$ & Static & No & Policies & Passive & $\begin{array}{l}\text { Control- and data-channel resource } \\
\text { optimization via spectrum division (SDR) }\end{array}$ \\
\hline$[3]$ & No & Custom & In-band & Custom & Yes & MPLS & Passive & WBN solution with custom SDN protocol \\
\hline [39] & Yes & Static & $\begin{array}{l}\text { Out-of-band } \\
\text { (add. NIC) }\end{array}$ & No & No & Manual & Wired & Experiments: simple flow redirection \\
\hline [11] & Yes & Static & In-band & $\begin{array}{l}\text { Extended- } \\
\text { OF }\end{array}$ & No & Shortest path & Passive & $\begin{array}{l}\text { Shortest path routing with bootstrapping } \\
\text { architecture }\end{array}$ \\
\hline [40] & Yes & Static & $\begin{array}{l}\text { Out-of-band } \\
\text { (add. WNIC) }\end{array}$ & OLSR & No & Assisted OLSR & No & Study on hybrid routing strategies \\
\hline [41] & Yes & $\begin{array}{l}\text { Static but extended- } \\
\text { OF monitoring }\end{array}$ & In-band & $\begin{array}{l}\text { Extended- } \\
\text { OF }\end{array}$ & No & $\begin{array}{l}\text { Interference, } \\
\text { Link-Load, CPU }\end{array}$ & No & $\begin{array}{l}\text { Dynamic load-balancing process due to } \\
\text { extended-OF monitoring }\end{array}$ \\
\hline
\end{tabular}

OF, OF is used; Wirel. int., Configuration and monitoring of WNIC; Ctl-Conn., Type of control-plane connection; Topo.-Disc., Topology discovery is addressed; M\&C, Modulation and coding is addressed; Routing, Type/goal of routing algorithm; Clients, Clients are monitored (active) or evaluated (passive); WNIC, Wireless Network Interface Controller.

protocol should be used for the wireless management. The edge interfaces of the WMN are afterwards exposed to the SDN controller as a simple switch.

The authors in [47] utilize the Open vSwitch $(\mathrm{OvS})$ in their nodes combined with a local controller (called second-tier controller) that handles the local status and configuration of wireless interfaces. The configuration is not exposed to the centralized controller but is situated on the distributed wireless nodes which prevents sophisticated SDWMN network applications.

Additional software can be deployed on both the controller device and the nodes. Forming a server-agent hierarchy, the wireless configuration is executed separately from the standard SDN mechanisms. This approach leaves the SDN protocol and controller unchanged. In [5], a custom server (Nagios) handles the topology, associations between stations, and monitors changes in order to adapt the network accordingly. A similar approach for the configuration of wireless interfaces is proposed in [34] called Ethanol. This framework defines a custom protocol between the controller and the wireless nodes based on XML-RPC. Detailed thoughts are given on which properties (observable or configurable, i.e. SSID, or number of associated clients) are needed for a joined operation of 802.11 and SDN. The authors sort these properties into logical entities.
The work in [48] describes a similar idea of a plugin for a wireless SDN controller to interact with 802.11 interfaces. They propose to use a REST API due its simplicity, the possibility to use HTTP for transportation, and the accordance with the YANG modeling language.

The Open Networking Foundation, founded as an industry consortium backing the development of $\mathrm{OF}$, has published a wireless transport SDN concept report in [49]. To control wireless links, the report suggests using combination of NETCONF and SSH. The so-called mediators translate generic functions residing at the controller to vendor specific commands for different equipments. A SDN capable software switch, for example the OvS, can be modified to send, receive and act on custom messages with the intent to allow for wireless configuration. Besides the mandatory control of the data-path (i.e. matches), the authors in [50] propose to add a link interface to the OvS allowing the controller to interact with the wireless interfaces in a media independent way, for example, to subscribe and receive events about link conditions (signal level) or to set interface parameters. In addition, the authors describe that a link media dependent interface is required on the switch to translate the generic messages into technology specific commands. While the authors describe several message formats, an example for the link media dependent interface is not provided. 
Extended OF messages to configure a radio module are also proposed in [38]. The authors make use of a Software-Defined Radio (SDR) on each wireless node. The extended OF protocol is used to set the frequency of the SDR. However, no details on these extensions are provided.

In general, the OF protocol was not designed to address the configuration of wireless interfaces. The protocol is not aware of the underlying physical technologies and should only handle configurations of data-paths for equivalent frame formats like IP, UDP or TCP. Nonetheless, it is possible to argue that OF is already the main protocol for the control-plane of wired SDN and an additional protocol dedicated to wireless adds complexity.

The authors in [32] found that since 802.11 implies a different addressing principle, the seamless integration has been shown challenging: when packets are forwarded over wireless links, it is necessary to manipulate the source and destination addresses of each packet as it traverses through the network. The proposed solution is to change the concerning addresses at each hop. This is taken into account by adding new actions to the flow-table that target this specific issue. The same issue with a similar solution of rewriting the source and destination at each hop is described in [22]. Ref. [45] extends the OF protocol capabilities with messages specifically dedicated to this issue. The authors in [51] describe that current SDN software components rely on point-to-point communication. To incorporate the point-to-multi-point case the authors suggest an entity which multiplexes multiple wireless links over a single wireless device making use of virtual LAN (VLAN) tags. The authors in [52] show that instead of conducting a rewrite at each hop, the 4-address-mode is sufficient for multi-hop forwarding with $\mathrm{OF}$ and 802.11.

Another interesting aspect is discussed in [21]. The authors describe that a separation of control- and data-plane already existed before the rise of $\mathrm{OF}$ in wireless networks. In fact, Control And Provisioning of Wireless Access Points (CAPWAP) is a protocol standardized by the IETF to centralize the control especially for 802.11 networks [53]. The authors describe that CAPWAP delivers control frames to a centralized controller similar to OF. The protocol is also mentioned in [32] and the authors briefly describe differences between CAPWAP and a wireless SDN-based solution. CAPWAP keeps several variables and states between the controller and the Access Point (AP) in sync; therefore, a significant amount of intelligence is still located on the AP. From a practical point of view, the authors argue that CAPWAP controllers are proprietary systems without implementing a proper northbound API and are therefore hard to extend.

The authors in [54] present an extension of the well-known mininet framework to include 802.11 using ns-3 similar to the work in [52].

\subsubsection{Open issues}

Two research directions for sophisticated control of wireless interfaces are discussed: either to enhance current SDN protocols like OF with wireless-related control and monitoring functions or to develop a new centralized protocol specifically dedicated to this use-case. Figure 1 shows these two architectures compared side by side. However, a clear comparison of these approaches does not exist at the moment.

If an additional control protocol for wireless interfaces is used, there is no common standard used at the moment. The variety of approaches ranges from XML-RPC, over HTTP and REST to NETCONF and SSH. In addition, there is no discussion of the control-plane for these additional protocols which is especially evident during the bootstrapping phase. One possibility is to use the same control-path already established for the OF protocol messages. Another possibility is to completely decouple the protocol and include a dedicated routing algorithm. Despite its undeniable similarity to the challenge of configuring distributed wireless interfaces, none of the current work has used or adapted CAPWAP to function as a wireless SDN interface control protocol.

A media independent message format for various wireless technologies has been identified as beneficial compared to transmitting for example 802.11 specific commands from the controller. For this approach, it is required that each wireless interface is able to translate these commands into technology specific actions. In addition, a mechanism for the wireless interface to announce their related capabilities to the controller should be evaluated.

\subsection{Bootstrapping, controller connection and topology discovery}

Using the acquired access to parameters of the wireless interfaces described in the last subsection the next important aspect for SDWMN is the behavior of nodes in case of initializing the network or adding new ones to it. This process is called bootstrapping and the main goal is that a SDWMN node establishes a connection with the controller by their own. At this point, network management applications can take over and deploy a desired configuration toward arbitrary goals. The terms in-band and out-of-band have become a common way to express the type of control channel in SDN. In the out-of-band case, the control channel is completely separated from the network that the switch is controlling through OF. In the in-band case, the control-path may overlap with the network that the switch controls. An in-band control leads to the principle that the switch needs to handle the controller connectivity by itself. To realize this, a set of default flow-rules can be implemented on each switch [55].

In the case of wireless networks, the bootstrapping process is more challenging and requires two phases. Before the SDN control channel connection can be established, a SDWMN interface needs to connect to one of its already connected neighbors or the controller itself. In contrast to wired SDN, where connectivity is simply determined by plugging in a 

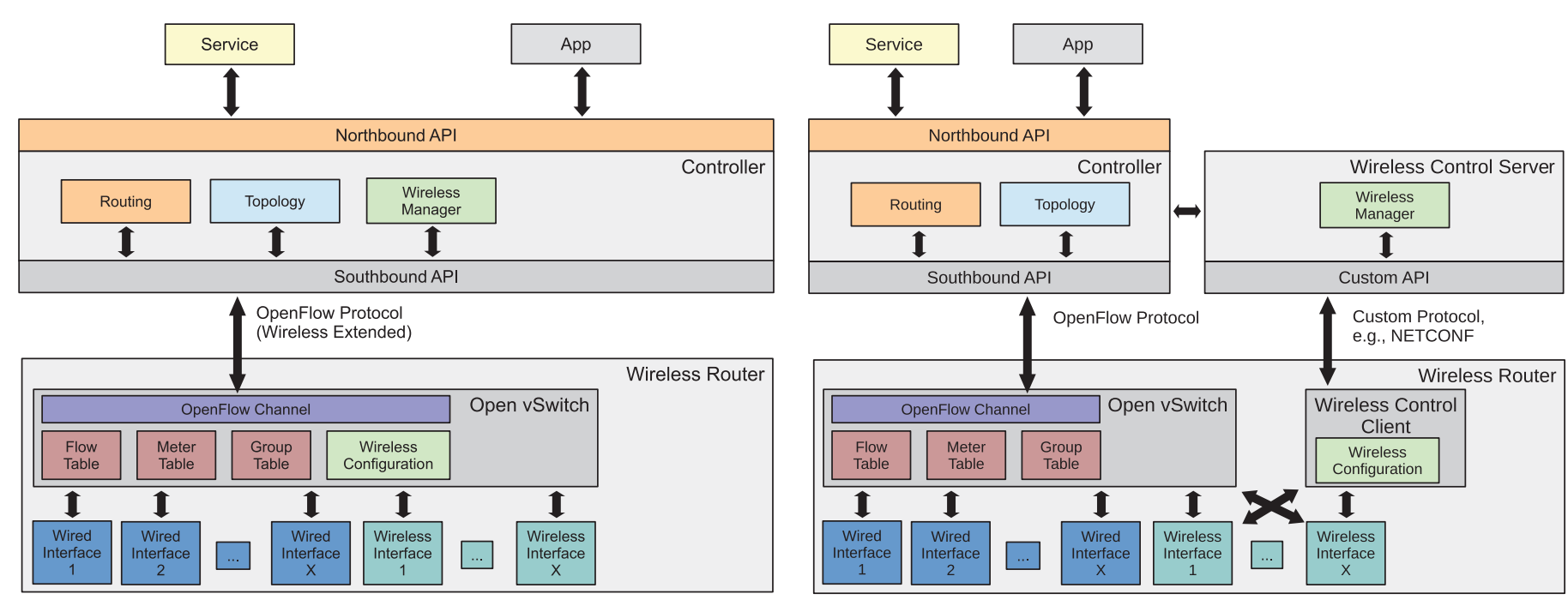

FIGURE 1. Different approaches to control wireless interfaces in SDWMN. On the left, extension of the OF protocol. On the right, custom control protocol for wireless interfaces.

cable and letting the auto-negotiation configure the link, there is no such default mechanism for wireless interfaces.

To have a complete representation of the network, the controller needs to be able to discover nodes, switches, links and host. This process is often referred to as topology discovery. Topology discovery in wired SDN networks makes use of the Link Layer Discovery Protocol (LLDP).

\subsubsection{Related work}

One possibility to address the aspect of bootstrapping and controller connection is to use a dedicated wired control network for SDWMN. While most consider it to be a reasonable conclusion that an additional wired infrastructure would not be favorable in SDWMN, for example [36, 50] utilize a wired control network for convenience. In [34], a wired infrastructure is already part of the project and is therefore used for transmitting control messages.

Out-of-band or in-band control channels imply both generic benefits as described in [55]. An out-of-band connection simplifies the switch implementation, is more reliable, since control traffic cannot interfere with data traffic, and provides advantages in terms of security, since not all machines in the data-path can snoop the control traffic. In-band control on the other hand has the benefit that no dedicated network interfaces are needed and therefore no dedicated network needs to be maintained.

The authors in [38] describe that current research in SDWMN can be classified into out-of-band or in-band connection for the control channel. The authors use a SDR board and argue that for wireless interfaces the in-band and out-ofband terms refer to different frequency-bands for data- and control-traffic. However, [5] is classified out-of-band in [38], despite the different SSIDs using the same interface and therefore the same frequency bands. The focus of their work is the evaluation of assigning sub-bands either to control or data traffic. The authors in [56] identify in-band connection in SDWMN as particularly challenging due to interference and possible power-saving mechanisms of the wireless interfaces. They propose to use a local controller handling this issue. This local controller synchronizes in-band with the global controller if the control channel is available. Similar ideas of using a local controller for handling the bootstrapping process are proposed in $[3,47]$.

The authors in [51] examine a wireless backhaul. The controller is attached in-band, with a preconfigured path through the use of one parent interface on each node, indicating the next hop toward the controller.

Establishing the control channel over a logically or physically separated wireless network implies several advantages and is therefore used for convenience for SDWMN. It can simplify the build-up and debugging because the control messages are transmitted in a dedicated network. It allows for hiding the control messages from the devices in the dataplane. It optimizes the throughput, since using a different physical interface for control traffic increases the available link capacity for data traffic. In addition, there might be an improved reliability because a node is still reachable for the controller even if the SDN data network is impaired, leaving the option of reconfiguration and recovery. However, the inband benefits described in [55] are even more evident for SDWMN. An interface port from the wired domain corresponds to a wireless interface. This wireless interface needs to be assigned to a dedicated channel and even be equipped with an additional directional antenna in the case of WBN which leads to an increase of CAPEX and complexity. 
One solution for the two-phase bootstrapping problem can be a distributed wireless mesh protocol which handles the physical connection of the interfaces. Afterwards, a SDN control channel can be established in-band using the configured wireless mesh interfaces. This solution is, for example, proposed in [22] where the authors use the OLSR protocol. In addition, the problem of assigning the controller IP address to the OF switches is solved by exploiting specific OLSR messages. However, this process involves a communication between the OLSR daemon and the OF daemon on the wireless nodes. The authors in [45] work with 802.11 dual-radio routers. In their approach, one wireless interface operates in the 802.11 s mesh mode and is solely used as a control channel interface. Therefore, this is an out-of-band approach. The authors in [5] argue that a connection is out-of-band if it is separated logically from the data traffic. In the wired context, this can be achieved through the use of different physical interfaces (physical segregation) or VLAN. Wireless interfaces do not support VLAN, hence the authors suggest separate SSID on top of the same physical interface (virtual segregation).

The discovery process in wired SDN-based networks is described in [57] using LLDP. Multi-hop topology discovery is identified as a major challenge for SDN wireless networks in Ref. [58]. The authors describe that broadcasting hello messages is a valid approach. This idea is similar to the LLDP protocol, however, LLDP has a one hop scope only [57].

The idea of SDN is to centralize most of the network intelligence at the controller. This idea is built on the principle that a reliable control channel to every device is available. In scenarios like the deployment of a campus or company 802.11 infrastructure networks, chances are high that a wired control network is available. This is different especially for WBN since the idea is to extend a network into areas without wired infrastructure. Based on the described work, Figure 2 visualizes three different possibilities to conduct a control connection in a SDWMN.

\subsubsection{Open issues}

Using the same channel for all wireless interfaces simplifies the bootstrapping process, since a maximum connectivity among nodes is reached but also minimizes the network-wide throughput. If an intelligent Channel Assignment (CA) algorithm is desired, which should be located on the controller, the initial bootstrapping and especially the fail-over scenario need to be evaluated additionally.

In-band control faces the challenge of changing medium conditions that can make the frequency of the control channel unreliable. In case there is no connectivity to the controller, the wireless nodes need to deal with this condition on their own. This demands a certain intelligence located on the wireless nodes. In wired SDN, this intelligence is already implemented in SDN switches, where a set of default rules on the switches conduct an initial in-band connection to the controller. Similar mechanisms are needed for wireless interfaces. However, the

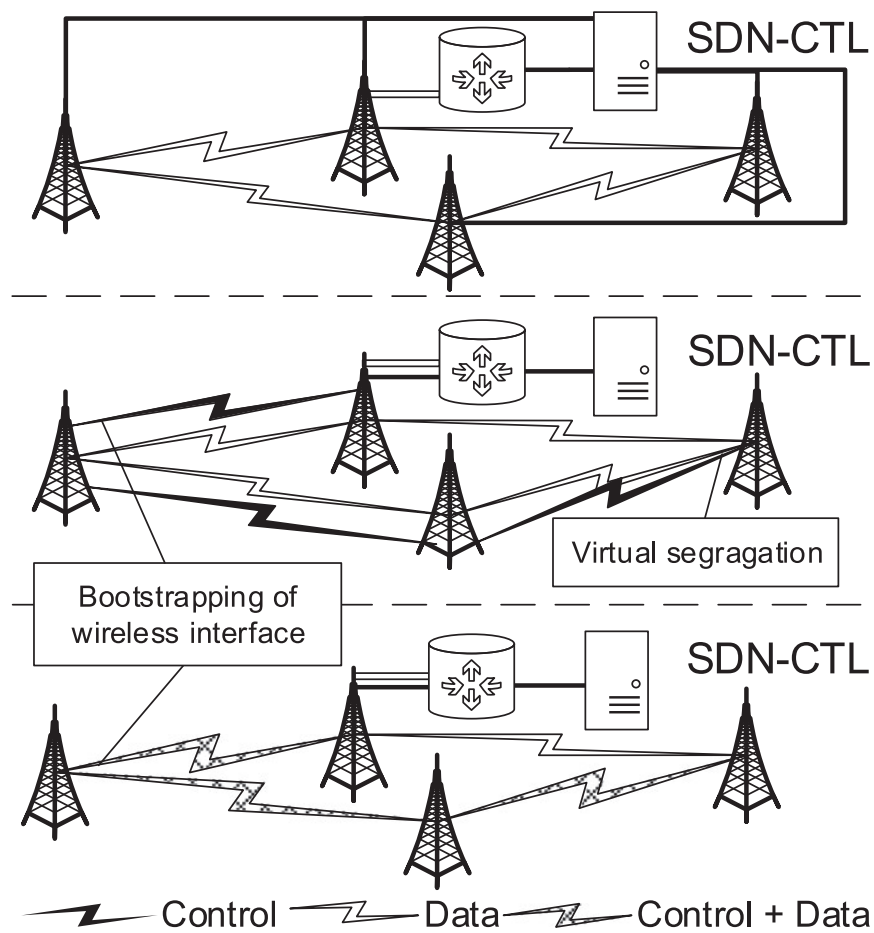

FIGURE 2. Bootstrapping and out-of-band or in-band connection for the control channel in SDWMN. Three different types. At the top, additional wired control network. In the middle, usage of two separate wireless interfaces (physical segregation) or different SSIDs or virtual interfaces (virtual segregation). At the bottom, classical inband connection.

usage of a distributed mesh protocol implementation on each device is not compliant with the general SDN principle. In our opinion, there are two different other possibilities which should be addressed in future research.

The first option is a default state for the wireless interfaces. This state implements an initial network connection to the controller or in case the node loses this connection. The benefit of this solution is its low complexity. However, defining this set of parameters independently from the wireless technology is challenging and requires additional thoughts in terms of wireless technology abstraction. A default state where nodes accept a new connection from an unknown node can be a security vulnerability. An authentication mechanism is therefore required. The channel used in the set of default parameters may be prone to interference. However, when the regulated Industrial, Scientific and Medical or Unlicensed National Information Infrastructure (U-NII) bands are used, chances are high that an initial connection is always possible.

The second option is that the nodes may use a dedicated scanning mechanism similar to the role of a client in an infrastructure network. On the one hand, this involves more complexity compared to a simple default state. On the other hand, issues like a jammed default control channel can be overcome. 


\subsection{Modulation and coding}

Compared to wired SDN where the capacity of a link is static and bounded to a few discrete values (i.e. $1 \mathrm{Gbps}, 10 \mathrm{Gbps}$ ) the situation for wireless SDN is different. After the bootstrapping phase described in the last subsection, the next important aspect is to handle the Modulation and Coding Scheme (MCS) on individual links. Modern wireless interfaces have the ability to choose between various different MCS to transmit a packet. An 802.11ac transmitter can choose between 10 different MCS from BPSK to 256-QAM. The optimal MCS depends on the Signal-to-Noise Ratio (SNR) which is influenced by two factors. First, an increasing distance of the wireless link decreases the signal at the receiver. Second, other participants on the same channel increase the noise which is sometimes noted as Signal-toInterference-plus-Noise Ratio.

\subsubsection{Related work}

The implementation of a MCS optimization algorithm requires access to relevant parameters mostly on the physical layer which is strongly related to the aspect representing wireless interfaces in SDN in general.

Extensive research has been conducted for MCS optimization algorithms especially in the context of 802.11. A comprehensive overview of existing algorithms is provided in Ref. [59]. The authors suggest a general classification of frame loss-based and SNR-based algorithms. The main issue for the former is that frame loss may appear due to channel fading or collision. According to [59], an algorithm should decrease the MCS only in response to channel degradation not in the case of collisions. The main issue for SNR-based MCS adaption is to precisely estimate the noise of the channel. The authors in [60] propose several guidelines for the design of MCS optimization algorithms like using probe packets or PHY metrics to access possible new rates.

An interesting discussion about current MCS algorithms is provided in [61]. The authors describe that these algorithms are distributed and work on a per link basis only. In the case of multi-hop WMN, choosing a MCS on one link can influence the optimum MCS on other links. In addition, MCS control has a strong relation to routing since the resulting data-rate determines the capacity of a link. The authors in [61] describe that along a chain, a more pessimistic MCS on the last link is better since possible re-transmission on this link are more expensive network-wide. The authors present a metric called expected transmission cost in multi-rate wireless networks to maximize the end-to-end throughput along a path.

In the case of SDWMN variable link characteristics have been identified as an issue [34]. However, no thoughts on MCS optimization are provided. The authors in [62] propose the idea of two different controllers in 802.11 SDN infrastructure networks. An additional controller located on the AP should be used to handle frequent and localized events close to the data-plane. Among others, one of the examples of such an event is to set bounding parameters for MCS optimization algorithms.

Figure 3 summarizes potential benefits for a centralized MCS optimization in SDWMN.

\subsubsection{Open issues}

If a WMN is used in the Mobile Ad hoc Network (MANET) context, MCS optimization needs to deal with changing conditions mainly due to the movements of nodes. In this case, a centralized algorithm may fail to react to fadings within an acceptable amount of time which is an interesting open research question for future SDWMN. In the case of WBN, channel conditions do not change frequently due to the static placement of the nodes and the usage of directional antennas. However, the lag between transmitting certain events to the controller and forwarding decisions back to the wireless switches can be above the requirements for certain use-cases. An example is Dynamic Frequency Selection, the requirement to avoid radar signal frequencies in the U-NII band.

For a centralized MCS optimization, an interesting open question is if single wireless links should be optimized independently or if it is beneficial to calibrate a multi-hop chain jointly. While the former can react better to local interference, the latter can capture dependencies between different MCS on different links.

A centralized algorithm can benefit from the global network view to conduct multi-hop optimization. Future research should investigate the possibility to implement flow-specific transmission parameters. If a new flow is set up, the controller may instruct the wireless nodes to use a certain MCS at different links for this particular flow. Specific QoS requirements like a decreased Packet Error Rate (PER) can be enforced using this technique. To decrease the interference

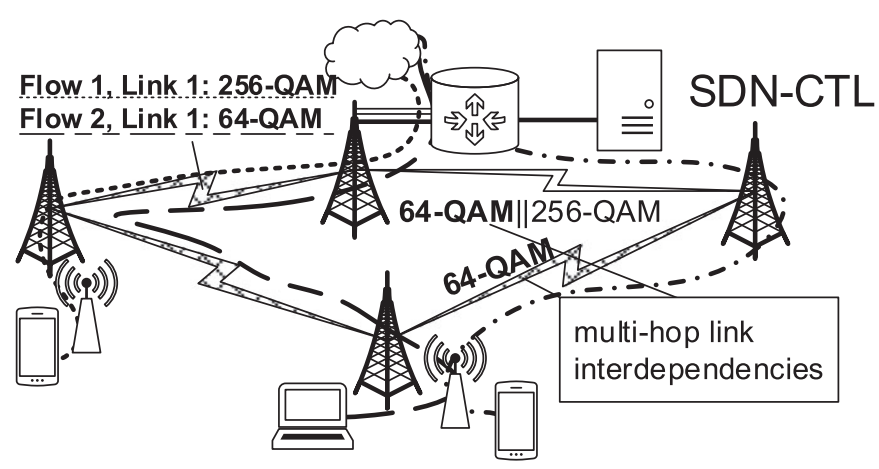

FIGURE 3. Benefits for a centralized MCS optimization in SDWMN: (1) Different flows may use different MCS depending on the path length. Flow 2 uses a 64-QAM to reduce the end-to-end PER. (2) Multi-hop links may have capacity or interference-related inter-dependencies among them. Using a 256-QAM is undesirable since there is no end-to-end throughput increase because of the second link which is bounded to a 64-QAM. 
level, a joint transmit power and MCS algorithm in SDWMN can be beneficial to decrease the inter-flow and intra-flow interference. Again, this joint approach benefits from a global network view at the controller.

\subsection{Routing and load-balancing}

After the successful bootstrapping phase and the determination of individual MCS parameters described in the last subsections, the next important aspect for SDWMN is a sophisticated routing approach. The availability of the current status of various devices and flows at the controller is one of the main advantages in SDN [63]. This increased knowledge provides the possibility to conduct an advanced forwarding, load-balancing and therefore better traffic engineering for all nodes in the network compared to distributed algorithms. A well-known traffic engineering example is the possibility to guarantee QoS based on user or application requirements. A key aspect to fulfill these requirements is to include them in the routing process. The programmable switches follow rules by the SDN controller and forward packets accordingly in order to determine what path the packets will take [63]. Traditional packet routing in WMN is decentralized, each node is responsible for its own forwarding decision [64]. Widely used protocols are OLSR, B.A.T.M.A.N. or Ad hoc On-Demand Distance Vector Routing Protocol (AODV). Compared to the SDN paradigm, every node has only partial knowledge of the network which makes traffic engineering challenging. However, especially in WMN, load-balancing is important since wireless nodes located near a gateway can become a bottleneck. The challenge of SDWMN is to make use of the enhanced traffic engineering capabilities. Figure 4 visualizes the idea of load-balancing in a SDWMN.

\subsubsection{Related work}

A comprehensive survey about traffic engineering techniques in wired SDN is provided in [63]. The authors provide an

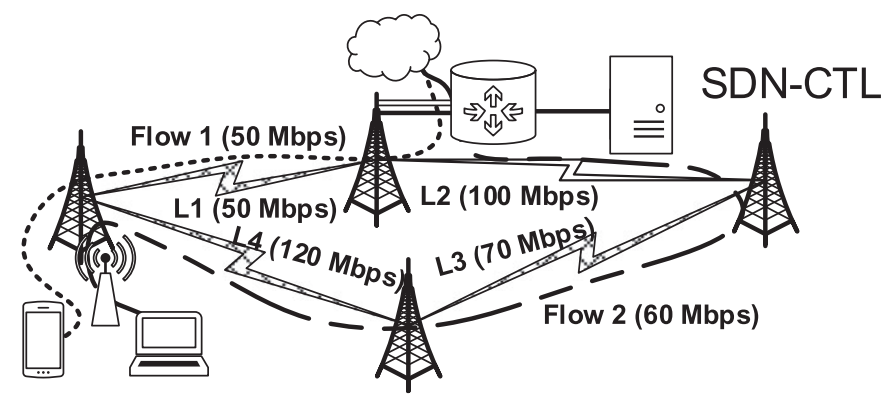

FIGURE 4. The aspect of load-balancing in SDWMN. To maximize the throughput for clients, two flows take different paths at the first possible hop. In this example, L1 is already saturated with flow 1 and therefore flow 2 is routed along an alternative (with an increased hop-count). overview of recent developments in flow management, fault tolerance, topology update and traffic analysis techniques. Their work shows a great interest in load-balancing for SDN.

Routing algorithms in WMN have been topic of extensive research. The authors in [64] provide a detailed classification and description of nearly 100 different WMN routing protocols.

In the context of MR-MC WMN, centralized routing protocols have been proposed even before the paradigm of SDN arose. Many of the proposed protocols are joint approaches solving the routing aspect in combination with related issues. The work in [65-67] tackle routing in conjunction with the CA problem. In general, these joint approaches have been identified as NP-hard and therefore approximation algorithms to find possible sub-optimal solutions in polynomial time are desired.

The authors in [68] describe centralized routing for small cell interconnections in the $5 \mathrm{G}$ context using a mmWave WMN. The authors suggest using a centralized controller which takes care of the configuration and routing without explicitly mentioning SDN. Their routing approach is based on multiple disjoint spanning trees rooted at the gateways. If a gateway link is congested, another spanning tree is used. In the following, we focus on work in the area of SDWMN for flow-based forwarding with load-balancing.

The authors in [39] show a very simple scenario using OF to change the path of a data-flow in a topology with four wireless nodes. However, the path change and the setup of the wireless links are conducted manually.

The goal of [40] is not to replace the distributed mesh protocols in WMN but to use an SDN approach to assist the routing process. The authors summarize that it is unfair to compare distributed routing protocols using in-band signaling with a centralized approach using out-of-band signaling. In their simulation-based study, the authors use OLSR with a limited hop-count for the messages. If the length of a path is greater than the limited hop-count, an out-of-band connected controller is used to conduct a shortest path computation. The authors show a small increase in throughput in their SDNassisted solution.

The authors in [35] compare distributed routing protocols like OLSR and B.A.T.M.A.N. with an OF-based solution in terms of throughput, jitter and loss. However, their comparison suffers from the fact that for the different protocols different 802.11 standards are used and that the SDN controller is connected out-of-band via Ethernet.

The authors in [69] study the problem of fault tolerance in the case that one wireless node breaks down. The importance of wireless nodes is calculated based on the number of direct neighbors and the controller is placed close to the most important ones. Afterwards, a minimum spanning tree is used to calculate the desired paths rooted from the controller.

The authors in [70] address the problem of energy consumption and security attacks on distributed WMN routing protocols by introducing a novel SDN-based approach. Their centralized routing strategy consists of three network 
applications: a hierarchical key management is complemented by calculating the reputation of a node and the energy consumption of different paths.

To conduct load-balancing in a SDWMN, the authors in [37] propose a hybrid approach. The authors use single-radio nodes and B.A.T.M.A.N. for node discovery and to set up a basic multi-hop connectivity. Load-balancing is afterwards realized on top of this WMN using OF. In addition, the authors argue that a monitoring client is needed on each switch without providing additional details on the requirements. Only very basic load-balancing tests are provided. The flow entry configuration is conducted manually without any automatism.

The authors in [41] propose a scheme to optimize the forwarding rules in SDWMN. The characteristics of their proposed scheme is 3-fold. During the bootstrapping phase, the SDN controller installs an initial set of rules based on routing policies like minimum-hop-count. In the second phase, statistics like wireless channel usage or the CPU load are gathered from all nodes. In the third phase, centralized optimization algorithms can conduct a flow reallocation if needed. Two comprehensive examples for load-balancing are provided based on the channel utilization and CPU load.

A three-phase routing approach for SDWMN is also proposed in [11]. The first stage is used to conduct an initial controller to switch connection by setting up basic routing. An adapted OLSR protocol located on the controller broadcasts information of directly connected switches. This information provides other switches with the ability to find a suitable path to the controller. In the second phase, a controller to switch path optimization based on the global network knowledge is conducted. In the last phase, the routing among the switches is optimized. An interesting contribution of this work is that the proposed routing scheme is implemented using the $\mathrm{OF}$ protocol without the need for additional software. However, the bootstrapping phase of wireless interfaces (to have a basic connection among the switches) is not discussed.

\subsubsection{Open issues}

For the forwarding and load-balancing aspect it is important to distinguish between different use-cases for a SDWMN. For MANET, a centralized SDN approach naturally implies certain drawbacks. If the topology of the network changes at a high frequency, the control traffic generated by the nodes and the controller may extend an undesirable threshold. In addition, centralized routing solutions imply an inherent lag, depending on the mobility of nodes, this lag can cause delayed routing updates. In addition, fault tolerance, scalability and the possibility for new nodes to join the network may be more important than the benefits of network wide traffic engineering. The applicability of SDN to MANET needs further investigation.

Hybrid or assisted routing solutions for SDWMN aim to incorporate benefits from both centralized and decentralized approaches. However, there is still a need for a clear investigation of potential benefits depending on the use-case. If an assisted solution uses an out-of-band control network with a dedicated interface on each node, a clear benefit compared to a pure decentralized solution using that extra interface is an unaddressed issue.

For WBN the situation is different. The placement of nodes is static, failures are rare and new nodes are added usually as an extension of an existing topology. In addition, the usage of multiple interfaces and different channels is usually mandatory for providing high throughput on the physical layer. The approaches in $[41,11]$ provide good ideas how to conduct a flow-based load-balancing at a centralized controller while still maintaining the idea of minimal direct touch of individual network components. Evaluating their approaches in large-scale SDWMN is desirable.

The already established approaches for centralized joint routing and CA in MR-MC WMN have not been used in the SDWMN domain. However, especially for WBN, an adaptation of these well-researched approaches seems worth considering.

\subsection{Client handling}

The idea of a communication network is to provide customers, users or clients access to different services like the Internet. Therefore, the next important aspect for SDWMN is to handle and optimize client access to the network. In a typical WMN, clients can connect to the same wireless interface used for meshing or to a dedicated access interface. This access interface typically uses the same technology but on a different channel. In WBN, a suitable access technology is deployed at all desired edges of the backhaul and 802.11 is among others a valid technology. In the case that multiple access interfaces exist in the same geographical area a control of the network load at these access interfaces is desired. For example, if multiple 802.11 AP exist but clients are crowded at a subset of these AP, load balancing among the AP can optimize the end-to-end throughput. In addition, including the access interfaces in the network management provides a holistic view and control for the operator. However, the main issue for an effective load-balancing of clients is that they associate or re-associate with an access interface on the basis of locally made decisions the infrastructure typically has no control over.

Besides the load-balancing of clients, additional services like mobility management, authentication, authorization and accounting or the dynamic reconfiguration of access channels are desired features for network operators. Commercial solutions for these service using a centralized controller exists. However, SDN has the potential to let operators run and program these services as a network application using vendorindependent equipment or even different technologies. 
Therefore, client handling is a common research field in the context of wireless SDN especially in the combination with 802.11. For the desired approach of SDWMN in the context of WBN, this field provides the chance to conduct optimization as close to the user as possible without influencing their equipment. An illustration of such an optimization is shown in Fig. 5.

\subsubsection{Related work}

The authors in [32] describe CloudMAC, an architecture where the management functionality of AP is virtualized. Every physical access interface is associated to one or more so-called VAP running on the controller. The MAC functionalities are split up between these two different instances. In fact, the main role of the physical AP is just to forward MAC frames. Physical AP and VAP are connected via an OF-based network. By reconfiguration of the intermediate flows, the controller determines which VAP instance is forwarded to which AP. Therefore, a VAP can be moved to another physical AP by a simple redirection of the traffic flow. The authors provide an example where one physical AP is turned off (due to energy savings) and the VAP including the associated station is moved without considerable packet loss.

A similar approach is proposed in [33]. Again the idea of VAP is inherited but with a different scope. The authors implement a master instance which instructs agents on each physical AP to spawn new VAP with a unique and mobile Basic service set identifier (BSSID) for each station. Therefore, each station is given the illusion that it connects to their own unique AP. The addresses of 802.11 beacons are modified to be unicast in this case and the traffic between the virtual $\mathrm{AP}$ and the controller is managed with $\mathrm{OF}$. The experiments conduct by the authors show that a client handoff between two physical AP can be achieved without affecting a HTTP download even when the handoff is realized among two different channels. Compared to [32], a significant amount of additional software is needed on each AP.

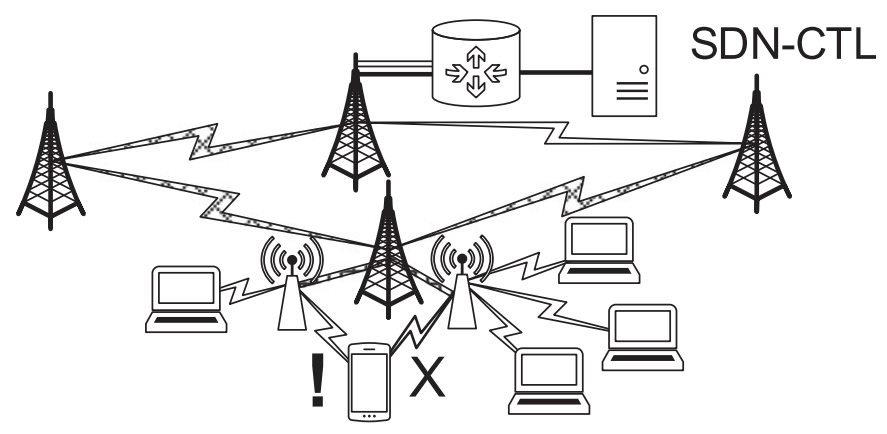

FIGURE 5. For an optimized end-to-end throughput devices should be distributed evenly (or even depending on their flows) to the access interfaces. In this example, the phone should be steered to the left access interface.
Both architectures in $[32,33]$ are using VAP to maintain handovers. The difference is that in [32] more than one client can connect to a VAP while exactly one VAP for every client is proposed in [33]. If the controller decides to move the VAP from one physical AP to another, all associated clients in [32] are moved. This can be sub-optimal if the clients are connected with different signal strengths.

The authors in $[71,72]$ assume that a mobile node has two wireless interfaces to associate with the network. One of them is used to maintain the connection to one AP and the other one is looking for an AP with better signal strength. If a better AP has been found, the mobile node connects to it while maintaining the connection to the old AP before the final handoff is conducted. The assumption that each client is equipped with multiple wireless interfaces of the same technology simplifies the handover process but is at the moment not the case for real-world equipment and demands additional intelligence on the client.

The authors in [73] argue that the 802.11 MAC layer can be decomposed into different functional blocks like association management or cryptography of frames. The idea of their work is to distribute these functionalities in the network. The authors suggest using the same BSSID, SSID and frequency on all AP simultaneously. Therefore, the network appears to the client as a single AP. All AP are connected with SDN enabled switches. The authors describe that loadbalancing in the network can be achieved by simply redirecting flows in the interconnection network.

Similar to [73], the authors in [74] describe that the MAC layer can be distributed in the network using the so-called lightweight AP which appear to clients as a single AP. However, the main difference is that there is no redirection of flows in the interconnection network. Instead, the authors suggest that every mobile client is linked to a home AP, usually the first AP the client connects to. If the client moves to another AP, a tunnel between the home AP and the new AP is initiated, bridging the data traffic. The authors state that the main benefit of this solution is that no redirection of flows in the interconnection network is required, which reduces the overhead. The idea, that a controller orchestrates the network to build a tunnel between two AP to prevent packet loss during a handover process, has also been discussed in $[75,76$, 77] before the concept of SDN emerged.

\subsubsection{Open issues}

To avoid bottlenecks for user terminals connecting to a SDWMN, a steering approach at the access interfaces is important. Current work focuses mainly on enterprise networks in campus like scenarios. Applying these approaches to SDWMN seems possible and desirable. Two different architectures, depending on the use of the wireless interfaces in the SDWMN, need to be evaluated. If the same wireless interface is used for backbone and access connections simultaneously, different virtual interfaces need to be used on top 
of the physical interface. While the approaches in [33, 32] support this architecture, possible inter-dependencies between the mesh and the access flows need to be investigated. In the case of WBN, it is common that different access and backhaul interfaces are used which simplifies the integration of the proposed load-balancing solutions into SDWMN.

The single AP solution proposed in $[73,74]$ demands more intelligence than just redirecting flows in the interconnection network. Every client is connected to more than one physical AP simultaneously, which multiplies the needed airtime for control frames. In addition, drop mechanisms at the AP are needed. An AP should drop data frames originated by a client if there is no active flow in the interconnection network for this client. Using the same channel on every AP lead to scalability issues due to higher interference among AP and clients.

For centralized handover control, additional thoughts on the underlying metrics and thresholds are needed to prevent constant association oscillation between AP. In addition, a well-defined northbound API for handover decisions can provide the ability to develop, evaluate and exchange this metrics using the concept of network applications.

\section{CONCLUSIONS}

In the wired SDN domain, the architecture of SDN converged to a common understanding among researchers. Therefore, even specific problems like controller placement, security or enhanced flow control are studied extensively [63]. In this work, we have presented the current state of SDN in the wireless domain focusing on the combination of SDN and WMN where there is at the moment no common ground for the architecture. We have shown that this merge is challenging, since the SDN domain relies on a centralized idea with minimal touch of individual network components while WMN are heavily dominated by distributed algorithms.

Wireless SDN requires access to parameters of the wireless interfaces. Transmission frequency, power, modulation and timings can be set by a local process on the wireless node, by an application running on the SDN controller, or by some joint mechanism where functionality is distributed between mesh node and controller. In order to integrate a (new) node into the SDWMN, a control channel between the mesh node and the controller needs to be established. This control channel can be in-band or out-of-band and should be established without operator involvement. This requires a topology discovery mechanism where new nodes are identified and integrated into the existing network, including handling of associated security threats and without using distributed mesh protocols on each SDN switch. While topology discovery is concerned with the identification of possible radio links, parametric optimization of individual links is the next step before a subset of possible links can be chosen for a routing process. These parameters include modulation and coding as well as timings and have a significant influence on link performance. Adapting MCS during network operation may require very fast algorithms and may be challenging for a centralized approach. A typical WMN supports more possible physical links than it can actually use. A mesh node may choose to use frequency $\mathrm{A}$ or $\mathrm{B}$ on one of its interfaces, connecting it to neighboring node $\mathrm{x}$ or $\mathrm{y}$, but not both. Path selection is an initial step toward routing, where additional aspects such as load balancing and fast rerouting may be taken into account. Finally, client access needs to be implemented in a way that makes optimal use of the scarce resource in a wireless network-the spectrum. While a clear split between access and backhaul frequencies allow simpler implementation, spectrum sharing can result in optimal use.

We are not aware of any holistic approach that addresses inter-dependencies between the different aspects. While each aspect of SDWMN can be investigated individually, they should take inter-dependencies into account. Modulation, link selection, routing, load-balancing, QoS provisioning are closely related in the wireless domain. In order to enable applications to address these inter-dependencies, the northbound interface of the wireless SDN controller needs to be standardized, in order to provide available networking parameters to the applications.

The authors in [8] summarize that the concept of SDN is not used in real-world WISP networks yet. However, in our opinion SDWMN provides a great opportunity for acting as a cost-efficient solution for terrestrial internet provisioning in rural areas and therefore demands further research activities taking into account the aspects presented in this work. The usage of Commercial Off-the-Shelf hardware, for example 802.11 transmitter and directional antennas, is already an established solution to decrease the CAPEX significantly. Centralized configuration and control aspects of SDN have the potential to significantly decrease the OPEX of WBN. There is no need for specialized staff in the field to conduct network maintenance in hard to reach areas, in fact, SDWMN has the potential to be a self-configuring carrier-grade system where network maintenance is just the process of exchanging defective hardware components.

\section{FUNDING}

The Federal Ministry of Education and Research of the Federal Republic of Germany [16KIS0332]. The authors alone are responsible for the content of this paper.

\section{REFERENCES}

[1] Kreutz, D., Ramos, F.M.V., Verissimo, P.E., Rothenberg, C.E., Azodolmolky, S. and Uhlig, S. (2015) Software-defined networking: a comprehensive survey. Proc. IEEE, 103, 14-76. 
[2] Doug, M., Jeff, D. and Pete, M. (2015) Software Defined Networking (SDN): Anatomy of OpenFlow. Volume I. Lulu Publishing Services, Raleigh, NC, USA.

[3] Hadzic, S., Niephaus, C., Aliu, O.G., Ghinea, G. and Kretschmer, M. (2015) Wireless Back-haul: a software defined network enabled wireless Back-haul network architecture for future 5G networks. IET Netw., 4, 287-295.

[4] Naudts, B., Kind, M., Westphal, F.-J., Verbrugge, S., Colle, D. and Pickavet, M. (2012) Techno-economic Analysis of Software Defined Networking as Architecture for the Virtualization of a MobileNetwork. 2012 European Workshop on Software Defined Networking (EWSDN), Darmstadt, Germany, October, pp. 67-72. IEEE.

[5] Dely, P., Kassler, A. and Bayer, N. (2011) OpenFlow for Wireless Mesh Networks. Proc. 20th Int. Conf. Comput. Commun. and Networks, Maui, Hawaii, July, pp. 1-6. IEEE.

[6] Gupta, P. and Kumar, P.R. (2000) The capacity of wireless networks. IEEE Trans. Inf. Theory, 46, 388-404.

[7] Mannweiler, C. and Schotten, H. (2014) Evaluating the Energy Balance of Solar-Powered Coordinated Wireless Backhaul Networks. 81st Veh. Technology Conf., Vancouver, Canada, September, pp. 1-5. IEEE.

[8] Hasan, S., Ben-David, Y., Bittman, M. and Raghavan, B. (2015) The Challenges of Scaling WISPs. Proc. Annu. Symp. Comput. Dev., New York, USA, December, pp. 3-11. ACM Press.

[9] Abujoda, A., Dietrich, D., Papadimitriou, P. and Sathiaseelan, A. (2015) Software-defined wireless mesh networks for internet access sharing. Comput. Netw., 93, 359-372.

[10] Hasan, S., Ben-David, Y., Scott, C., Brewer, E. and Shenker, S. (2013) Enhancing Rural Connectivity with Software Defined Networks. Proc. 3rd ACM Symp. Comput. Dev., New York, USA, January 1. ACM Press.

[11] Patil, P. (2016) Algorithms and techniques for transitioning to software defined networks. Phd Thesis, Vanderbilt University.

[12] Masoudi, R. and Ghaffari, A. (2016) Software defined networks: a survey. J. Netw. Comput. Appl., 67, 1-25.

[13] Akyildiz, I. and Wang, X. (2005) A survey on wireless mesh networks. IEEE Commun. Mag., 43, 23-30.

[14] Misra, S., Misra, S.C. and Woungang, I. (eds.) (2009) Guide to Wireless Mesh Networks Computer Communications and Networks. Springer, London.

[15] Pathak, P.H. and Dutta, R. (2011) A survey of network design problems and joint design approaches in wireless mesh networks. IEEE Commun. Surv. Tutor., 13, 396-428.

[16] Hu, F., Hao, Q. and Bao, K. (2014) A survey on softwaredefined network and OpenFlow: from concept to implementation. IEEE Commun. Surv. Tutor., 16, 2181-2206.

[17] Hakiri, A., Gokhale, A., Berthou, P., Schmidt, D.C. and Gayraud, T. (2014) Software-defined networking: challenges and research opportunities for future internet. Comput. Netw., 75, 453-471.

[18] Jagadeesan, N.A. and Krishnamachari, B. (2014) Softwaredefined networking paradigms in wireless networks: a survey. ACM Comput. Surv., 47, 1-11.
[19] Kobo, H.I., Abu-Mahfouz, A.M. and Hancke, G.P. (2017) A survey on software-defined wireless sensor networks: challenges and design requirements. IEEE Access, 5, 1872-1899.

[20] Haque, I.T. and Abu-Ghazaleh, N. (2016) Wireless software defined networking: a survey and taxonomy. IEEE Commun. Surv. Tutor., 18, 2713-2737.

[21] Bernardos, C.J., De La Oliva, A., Serrano, P., Banchs, A., Contreras, L.M., Jin, H. and Zuniga, J.C. (2014) An architecture for software defined wireless networking. IEEE Wirel. Commun., 21, 52-61.

[22] Detti, A., Pisa, C., Salsano, S. and Blefari-Melazzi, N. (2013) Wireless Mesh Software Defined Networks (wmSDN). 9th Int. Conf. Wirel. Mob. Comput. Netw. Commun., Lyon, France, October, pp. 89-95. IEEE.

[23] Tomovic, S., Yoshigoe, K., Maljevic, I., Pejanovic-Djurisic, M. and Radusinovic, I. (2014) SDN-Based Concept of QoS Aware Heterogeneous Wireless Network Operation. Telecommunications Forum Telfor (TELFOR), Belgrade, Serbia, November, pp. 27-30. IEEE.

[24] Ali-Ahmad, H., Cicconetti, C., De La Oliva, A., Mancuso, V., Reddy Sama, M., Seite, P. and Shanmugalingam, S. (2013) An SDN-Based Network Architecture for Extremely Dense Wireless Networks. IEEE SDN Future Networks and Services, Trento, Italy, January, pp. 1-7. IEEE.

[25] Pentikousis, K., Wang, Y. and Hu, W. (2013) Mobileflow: toward software-defined mobile networks. IEEE Commun. Mag., 51, 44-53.

[26] Jin, X., Li, L.E., Vanbever, L. and Rexford, J. (2013) Softcell: Scalable and Flexible Cellular Core Network Architecture. Proc. 9th ACM Conf. Emerg. Netw. Exp. Technol., New York, USA, December, pp. 163-174. ACM Press.

[27] Nguyen, V.-G., Do, T.-X. and Kim, Y. (2016) SDN and virtualization-based LTE mobile network architectures: a comprehensive survey. Wirel. Pers. Commun., 86, 1401-1438.

[28] Yang, M., Li, Y., Jin, D., Zeng, L., Wu, X. and Vasilakos, A. V. (2015) Software-defined and virtualized future mobile and wireless networks: a survey. Mob. Netw. Appl., 20, 4-18.

[29] Yap, K.-k., Kobayashi, M., Sherwood, R., Huang, T.-y., Chan, M., Handigol, N. and Mckeown, N. (2010) OpenRoads: empowering research in mobile networks. ACM SIGCOMM Comput. Commun. Rev., 40, 125-126.

[30] Rob, S., Gibb, G., Yap, K., Appenzeller, G., Casado, M., McKeown, N. and Parulkar, G. (2009) Flowvisor: A Network Virtualization Layer. Technical Report. OpenFlow Switch Consortium, Stanford, CA, USA.

[31] Fontes, R. d. R., Mahfoudi, M., Dabbous, W., Turletti, T. and Rothenberg, C. (2017) How far can we go? towards realistic software-defined wireless networking experiments. Comput. J., 1, 1-14.

[32] Dely, P., Vestin, J., Kassler, A., Bayer, N., Einsiedler, H. and Peylo, C. (2012) CloudMAC-An OpenFlow based Architecture for 802.11 MAC Layer Processing in the Cloud. Globecom Work, Anaheim, CA, USA, December, pp. 186-191. IEEE.

[33] Suresh, L., Schulz-Zander, J., Merz, R., Feldmann, A. and Vazao, T. (2012) Towards Programmable Enterprise WLANS 
with Odin. Proc. 1st Work. Hot Top. Softw. Defined Networks, New York, USA, August 1-15. ACM Press.

[34] Moura, H., Bessa, G.V.C., Vieira, M.A.M. and Macedo, D.F. (2015) Ethanol: Software Defined Networking for 802.11 Wireless Networks. IFIP/IEEE Int. Symp. Integr. Netw. Manag., 1, 388-396.

[35] Chung, J., Gonzalez, G., Armuelles, I., Robles, T., Alcarria, R. and Morales, A. (2012) Characterizing the Multimedia Service Capacity of Wireless Mesh Networks for Rural Communities. 8th Int. Conf. Wirel. Mob. Comput. Netw. Commun., Barcelona, Spain, October, pp. 628-635. IEEE.

[36] Chung, J., Gonzalez, G., Armuelles, I., Robles, T., Alcarria, R. and Morales, A. (2013) Experiences and challenges in deploying OpenFlow over real wireless mesh networks. IEEE Lat. Am. Trans., 11, 955-961.

[37] Yang, F., Gondi, V., Hallstrom, J.O., Wang, K.-C. and Eidson, G. (2014) OpenFlow-based Load Balancing for Wireless Mesh Infrastructure. IEEE 11th Consum. Commun. Netw. Conf., Las Vegas, NV, USA, January, pp. 444-449. IEEE.

[38] Huang, H., Li, P., Guo, S. and Zhuang, W. (2015) Softwaredefined wireless mesh networks: architecture and traffic orchestration. IEEE Netw., 29, 24-30.

[39] Yang, H., Chen, B. and Fu, P. (2015) Openflow-based Load Balancing for Wireless Mesh Network. Cloud Computing and Security. Lecture Notes in Computer Science, 9483.

[40] Labraoui, M., Boc, M.M. and Fladenmuller, A. (2016) Software Defined Networking-Assisted Routing in Wireless Mesh Networks. Int. Wirel. Commun. Mob. Comput. Conf., Paphos, Cyprus, September, pp. 377-382. IEEE.

[41] Betzler, A., Quer, F., Camps-mur, D., Demirkolt, I. and Garcia-villegast, E. (2016) On the Benefits of Wireless SDN in Networks of Constrained Edge Devices. Eur. Conf. Networks Commun., Athens, Greece, June, pp. 1-5. IEEE.

[42] Kretschmer, M., Horstmann, T., Batroff, P., Rademacher, M. and Ghinea, G. (2012) Link Calibration and Property Estimation in Self-managed Wireless Back-haul Networks. 18th Asia-Pacific Conf. Commun., Jeju Island, Korea, October, pp. 232-237. IEEE.

[43] Rademacher, M. (2015) Performance Estimation and Optimization of the IEEE802.11 MAC Layer for Long Distance Point-to-Point Links. Technical Report. Hochschule Bonn-Rhein-Sieg, St. Augustin, Germany.

[44] Rademacher, M., Kessel, M. and Jonas, K. (2016) Experimental Results for the Propagation of Outdoor IEEE802.11 Links. VDE ITG-Fachbericht Mobilkommunikation, 22.

[45] Nascimento, V., Moraes, M., Gomes, R., Pinheiro, B., Abelem, A., Borges, V.C.M., Cardoso, K.V. and Cerqueira, E. (2014) Filling the Gap Between Software Defined Networking and Wireless Mesh Networks. 10th Int. Conf. Netw. Serv. Manag. Work., Rio de Janeiro, Brazil, November, pp. 451-454. IEEE.

[46] Seppanen, K., Kilpi, J. and Suihko, T. (2015) Integrating WMN Based Mobile Backhaul with SDN Control. Lecture Notes Institute for Computer Science Social Telecommunications Engineerirng (LNICST), pp. 222-233. Springer, New York, USA.
[47] Mihailescu, M., Nguyen, H. and Webb, M.R. (2015) Enhancing Wireless Communications with Software Defined Networking. Mil. Commun. Inf. Syst. Conf., Cracow, Poland, November, pp. 1-6. IEEE.

[48] Nunez-Martinez, J., Baranda, J., Pascual, I. and ManguesBafalluy, J. (2016) WiseHAUL: An SDN-empowered Wireless Small Cell Backhaul Testbed. 17th Int. Symp. A World Wireless, Mob. Multimed. Networks, Coimbra, Portugal, June, pp. 1-3. IEEE.

[49] Representatives from the wireless transport eco system (2016) Wireless Transport SDN Proof of Concept 2 Detailed Report. Technical Report. Open Networking Foundation, Palo Alto.

[50] Guimaraes, C., Corujo, D. and Aguiar, R.L. (2014) Enhancing Openflow with Media Independent Management capabilities. IEEE Int. Conf. Commun., Sydney, Australia, June, pp. 2995-00. IEEE.

[51] Hurtado-Borras, A., Pala-Sole, J., Camps-Mur, D. and SallentRibes, S. (2015) SDN Wireless Backhauling for Small Cells. 2015 IEEE Int. Conf. Commun., London, UK, June, pp. 3897-02. IEEE.

[52] Rademacher, M., Siebertz, F., Schlebusch, M. and Jonas, K. (2016) Experiments with OpenFlow and IEEE802.11 Point-toPoint Links in a WMN. Twelfth Int. Conf. Wirel. Mob. Commun. (ICWMC), pp. 99-105. IARIA, Barcelona, Spain.

[53] Calhoun, P., Montemurro, M. and Stanley, D. (2009). Control and Provisioning of Wireless Access Points (CAPWAP) Protocol Specification. RFC 5415 (Proposed Standard).

[54] Fontes, R.R., Afzal, S., Brito, S.H.B., Santos, M.A.S. and Rothenberg, C.E. (2015) Mininet-WiFi: Emulating SoftwareDefined Wireless Networks. 2015 11th Int. Conf. Netw. Serv. Manag., Barcelona, Spain, November, pp. 384-389. IEEE.

[55] vSwitch, O. Design.rst. https://github.com/openvswitch/ovs. (30/10/2016, 28/11/2016).

[56] Lee, J., Uddin, M., Tourrilhes, J., Sen, S., Banerjee, S., Arndt, M., Kim, K.-h. and Nadeem, T. (2014) meSDN. Proc. 5th Int. Work. Mob. Cloud Comput. Serv., New York, USA, June, pp. 7-14. ACM Press.

[57] Ochoa Aday, L., Cervello Pastor, C. and Fernandez, A. (2015) Current Trends of Topology Discovery in OpenFlow-based Software Defined Networks. Departament d'Enginyeria Telemàtica-Reports de recerca. Universitat Politècnica de Catalunya, Barcelona, Spain.

[58] Chaudet, C. and Haddad, Y. (2013) Wireless Software Defined Networks: Challenges and Opportunities. IEEE Int. Conf. Microwaves, Commun. Antennas Electron. Syst., Tel Aviv, Israel, October, pp. 1-5. IEEE.

[59] Biaz, S. and Wu, S. (2008) Rate Adaptation Algorithms for IEEE 802.11 Networks: A Survey and Comparison. IEEE Symp. Comput. Commun., Marrakech, Morocco, July, pp. 130-136. IEEE.

[60] Wong, S.H., Yang, H., Lu, S. and Bharghavan, V. (2006) Robust Rate Adaptation for 802.11 Wireless Networks. Proc. 12th Annual Int. Conf. Mobile Computing and Networking, Los Angeles, CA, USA, September, pp. 146-157. ACMACM.

[61] Kim, T.-S., Jakllari, G., Krishnamurthy, S.V. and Faloutsos, M. (2013) An integrated routing and rate adaptation framework for 
multi-rate multi-hop wireless networks. Wirel. Netw., 19, 985-1003.

[62] Schulz-Zander, J., Sarrar, N. and Schmid, S. (2014) Towards a Scalable and Near-sighted Control Plane Architecture for WiFi SDNs. Proc. 3rd Work. Hot Top. Softw. Defined Netw., New York, USA, August, pp. 217-218. ACM Press.

[63] Akyildiz, I.F., Lee, A., Wang, P., Luo, M. and Chou, W. (2014) A roadmap for traffic engineering in SDN-OpenFlow networks. Comput. Netw., 71, 1-30.

[64] Alotaibi, E. and Mukherjee, B. (2012) A survey on routing algorithms for wireless ad-hoc and mesh networks. Comput. Netw., 56, 940-965.

[65] Alicherry, M., Bhatia, R. and Li, L.E. (2006) Joint channel assignment and routing for throughput optimization in multiradio wireless mesh networks. IEEE J. Sel. Areas Commun., 24, 1960-1971.

[66] Raniwala, A., Gopalan, K. and Chiueh, T.-c. (2004) Centralized channel assignment and routing algorithms for multi-channel wireless mesh networks. ACM SIGMOBILE Mob. Comput. Commun. Rev., 8, 50.

[67] Tang, J., Xue, G. and Zhang, W. (2005) Interference-Aware Topology Control and QoS Routing in Multi-channel Wireless Mesh Networks. Proc. 6th ACM Int. Symp. Mob. Ad Hoc Netw. Comput., New York, USA, May 6-8. ACM Press.

[68] Seppanen, K., Kilpi, J., Paananen, J., Suihko, T., Wainio, P. and Kapanen, J. (2016) Multipath routing for mmWave WMN backhaul. IEEE Int. Conf. Commun. Work, Kuala Lumpur, Malaysia, May, pp. 246-253. IEEE.

[69] Peng, Y., Gong, X., Guo, L. and Kong, D. (2016) A survivability routing mechanism in SDN enabled wireless mesh networks: design and evaluation. China Commun., 13, 32-38.
[70] Lin, H., Hu, J., Xu, L., Tian, Y., Liu, L. and Blakeway, S. (2016) A trustworthy and energy-aware routing protocol in software-defined wireless mesh networks. Comput. Electr. Eng., 0, 1-13.

[71] Ivov, E. and Noel, T. (2005) Soft handovers over $802.1 \mathrm{lb}$ with multiple interfaces. 2nd Int. Symposium on Wireless Communication Systems, 2005. Siena, Italy, September, pp. 549-554. IEEE.

[72] Salsano, S., Polidoro, A., Mingardi, C., Niccolini, S. and Veltri, L. (2008) Sip-based mobility management in next generation networks. IEEE Wirel. Commun., 15, 92-99.

[73] Zhao, D., Zhu, M. and Xu, M. (2014) SDWLAN: A Flexible Architecture of Enterprise WLAN for Client-unaware Fast AP Handoff. In Fifth Int. Conf. Comput. Commun. Netw. Technol., Hefei, China, July, pp. 1-6. IEEE.

[74] Sen, A. and Sivalingam, K.M. (2015) An SDN Framework for Seamless Mobility in Enterprise WLANs. 26th Annu. Int. Symp. Pers. Indoor, Mob. Radio Commun., Hong Kong, August, pp. 1985-90. IEEE.

[75] Huang, R., Zhang, C. and Fang, Y. (2007) A Mobility Management Scheme for Wireless Mesh Networks. Global Telecommunications Conf., 2007. (GLOBECOM'07). IEEE, Washington, DC, USA, November, pp. 5092-96. IEEE.

[76] Li, Y. and Chen, R. (2011) Design and performance analysis of mobility management schemes based on pointer forwarding for wireless mesh networks. IEEE Trans. Mob. Comput., 10, 349-361.

[77] Wang, H., Huang, Q., Xia, Y., Wu, Y. and Yuan, Y. (2007) A Network-based Local Mobility Management Scheme for Wireless Mesh Networks. Wireless Communications and Networking Conf., 2007 (WCNC2007), Hong Kong, March, pp. 3792-97. IEEE. 